\title{
The cosmic ray content of superbubbles
}

\author{
Thibault Vieu, ${ }^{a, *}$ Stefano Gabici ${ }^{a}$ and Vincent Tatischeff ${ }^{b}$ \\ ${ }^{a}$ Université de Paris, CNRS, Astroparticule et Cosmologie, \\ F-75013 Paris, France \\ ${ }^{b}$ Université Paris-Saclay, CNRS/IN2P3, IJCLab, \\ 91405 Orsay, France \\ E-mail: vieu@apc.in2p3.fr
}

\begin{abstract}
Although most massive stars are thought to live inside clusters giving rise to galactic-scale superbubbles, a complete model of cosmic ray production in these objects is still missing in the literature. We present an attempt to model particles acceleration in these systems including all the relevant physical processes. The acceleration mechanisms taking place in superbubbles differ from that occurring at isolated supernova remnants in several aspects. The presence of a magnetized shell is expected to efficiently confine the particles, which may therefore experience successive reaccelerations at supernovae shocks. The medium is also though to be highly turbulent, which hardens the particle spectra and makes shock acceleration nonlinear. Spectra are further shaped by losses, stellar winds, and the leakage of the interstellar cosmic rays through the superbubble shell. We discuss typical superbubble spectra and detail the time-dependent emission of these objects.
\end{abstract}

$37^{\text {th }}$ International Cosmic Ray Conference (ICRC 2021)

July 12th - 23rd, 2021

Online - Berlin, Germany

\footnotetext{
${ }^{*}$ Presenter
} 


\section{Introduction}

Superbubbles are expanding cavities carved inside dense molecular clouds by the feedback of clustered massive stars. It is thought that most massive stars are actually born in either compact or loose associations, in which they spend most of their lives until they explode as supernovae [1]. On the other hand, massive stars have long been thought to be the major sources of galactic cosmic rays, in particular by diffusive shock acceleration at wind termination shocks and supernova remnants. In the recent years, there have been indeed several observations of non-thermal emission in star forming regions and superbubbles [2-6]. Early models of particle acceleration inside clusters have been developed by Bykov and collaborators [7], who assumed that superbubble environments were characterised by a stochastic ensemble of secondary shock waves, which is in general only expected in the first few million years of the cluster life. More recently, Ferrand and Marcowith [8] computed the average cosmic ray spectra in evolved superbubbles, accounting for the reacceleration at supernova remnant shocks as well as the diffusive acceleration in weak turbulence. In the present work, we seek to develop a self-consistent model from first principles, including all the relevant physical ingredients, namely stellar winds, supernova remnants, turbulence, losses, in a realistic time-dependent model of acceleration accounting for the backreaction of the particles on the shocks and on the turbulence.

\section{A model of particle acceleration in superbubbles}

\subsection{Bubble structure}

Let us consider a cluster containing hundreds of $8-150 M_{\odot}$ stars distributed according to a Salpeter initial mass function. Numerical fits of stellar evolution models [9] allow to estimate the lifetime of each star and thus the time of supernova explosions. Assuming that all stars are born at the same time, we run a Monte-Carlo to simulate typical clusters. The winds and supernovae impart a strong energy input in the surrounding medium, which is swept-up and accumulated in an expanding shell, leaving behind a low-density cavity called a superbubble. The radius of the superbubble is determined from thermodynamic considerations as [10]:

$$
R_{b}(t) \approx R_{0} N_{*}^{1 / 5}\left(\frac{t}{\mathrm{Myr}}\right)^{3 / 5},
$$

where $N_{*}$ is the initial number of massive stars in the cluster and $R_{0} \approx 10 \mathrm{pc}$ is estimated from observations. Eventually, the evolution of the interior density and temperature is driven by thermal conduction and mass evaporation [10,11].

\subsection{Particle acceleration at shocks}

We assume that the outflows of massive stars produce strong wind termination shocks. The wind mechanical power $\mathcal{P}_{w}$ in the main sequence and in the Wolf-Rayet phase is computed from fits of stellar evolution models [12]. Because winds are only expected to accelerate a moderate density of particles, the test-particle regime holds and we describe their contribution as a background $p^{-4}$ 
injection modulated by an exponential cutoff at a momentum $p_{w}[13]$ :

$$
q_{w}(p)=\frac{\eta_{w} p^{-4} e^{-p / p_{w}}}{4 \pi \mathcal{N}_{w}} \sum_{i=1}^{N(t)} \mathcal{P}_{w}\left(M_{i}\right),
$$

where $N(t)$ is the number of massive stars remaining in the cluster at time $t$ and $\mathcal{N}_{w}$ is a normalisation constant ensuring that a fraction $\eta_{w}(\approx 10 \%)$ of the wind energy is transferred into cosmic rays.

When a star explodes in a supernova at the end of its life, a strong shock is launched inside the superbubble. Not only this shock injects "fresh" cosmic rays from the thermal bath, but it also reaccelerates preexisting particles confined within the bubble upstream of the shock. Because the cosmic ray energy density in bubbles can be comparable to that of supernova shocks, it is mandatory to describe the reacceleration process accounting for the nonlinear backreaction of the preexisting particles. In order to properly account for this reacceleration mechanism, we updated the model of Blasi [14], including the thermal leakage injection mechanism [15] as well as the resonant excitation of waves [16, 17]. The nonlinear reacceleration model will be detailed in [18].

\subsection{Stochastic reacceleration in turbulence}

When particles diffuse inside the superbubble, they interact with stochastic magnetic perturbations, which gives rise to a diffusive acceleration in momentum space. It can be shown from first principles that the momentum diffusion coefficient reads [19]:

$$
D_{p}=\frac{\pi^{2} Z^{2} e^{2} v_{A}^{2}}{v} \int_{Z e B / p}^{\infty} \frac{\mathrm{d} k}{k}\left(1-\left(\frac{Z e B}{k p}\right)^{2}\right) W(k),
$$

where $W$ is the turbulence spectrum, $Z e$ is the charge of the particle (although the present work focuses on protons with $Z=1), v_{A}$ is the Alfvén velocity, $v$ the velocity of the particles and $B$ the magnetic field. The spectrum of the turbulence is determined by the equation describing the turbulence cascade, which can be written in the stationary Kolmogorov regime as:

$$
\partial_{k}\left(\frac{a}{\sqrt{\rho}} k^{5 / 2} W^{3 / 2}\right)=-\Gamma(k) W+S \delta\left(k-k_{0}\right),
$$

where $a \approx 0.8$ is a numerical constant determined from experiments or simulations (e.g. [20]), $\rho$ is the gas density, $2 \pi / k_{0}$ is the largest turbulence scale, $S$ is the injection at the largest scale and $\Gamma$ is the damping rate. We assume that the stars convert a fraction $\eta_{T}$ of their mechanical energy into hydromagnetic waves, such that the source term reads (e.g. [21]):

$$
S=\frac{\eta_{T}}{V_{S B}(t)} \mathcal{P}_{t o t}
$$

where $\mathcal{P}_{\text {tot }}$ is the total mechanical power accounting for both winds and supernovae and $V_{S B}$ is the volume of the superbubble. The damping rate $\Gamma$ is obtained after writing the energy balance between the waves and the particles as (e.g. [22]):

$$
\Gamma(k)=\frac{8 \pi^{3} Z^{2} e^{2} v_{A}^{2}}{k} \int_{Z e B / k} \mathrm{~d} p p f(p),
$$

and the momentum diffusion coefficient can be determined self-consistently as function of the particle distribution function, making the problem nonlinear. 


\begin{tabular}{lcc}
\hline Initial number of massive stars & $N_{*}$ & $100,500 \mathrm{or} 1000$ \\
Normalised radius & $R_{0}$ & $10 \mathrm{pc}$ \\
Density of the interstellar medium & $n_{I S M}$ & $100 \mathrm{~cm}^{-3}$ \\
Large scale magnetic field & $B_{0}$ & $10 \mu \mathrm{G}$ \\
Efficiency of turbulence generation & $\eta_{T}$ & $1 \%$ or $30 \%$ \\
Relaxation time of the turbulence & $\tau_{T}$ & $1 \mathrm{Myr}$ \\
Largest turbulent scale & $\lambda$ & $10 \mathrm{pc}$ \\
Injection parameter of supernova shocks & $\alpha$ & 3.5 \\
Acceleration efficiency at winds & $\eta_{w}$ & $10 \%$ \\
Injection momentum at winds & $p_{0}$ & $5 \mathrm{MeV}$ \\
Maximum energy at winds & $p_{w}$ & $10^{5} \mathrm{GeV}$ \\
\hline
\end{tabular}

Table 1: Exhaustive list of parameters of the model.

\subsection{Transport of particles in superbubbles}

Not only the turbulence reaccelerates cosmic rays in the superbubble, it also induces a diffusive escape. It can be shown from first principles that the spatial and momentum diffusion coefficients obey in the Kolmogorov regime $D_{x} D_{p} \approx 0.2 v_{A}^{2} p^{2}$. Then from the momentum diffusion coefficient we obtain the escape time as $\tau=R_{b}^{2} /\left(6 D_{x}\right)$. Eventually, the complete transport equation of cosmic rays in the superbubble reads, in term of the average particle energy spectrum $n=f / V_{S B}$ :

$$
\partial_{t} n=-\frac{n}{\tau}+\frac{1}{p^{2}} \partial_{p}\left(p^{2} D_{p} \partial_{p} n\right)+\frac{1}{p^{2}} \partial_{p}\left(p^{2}\left(\frac{3 \epsilon(p)}{5 v t}+\left.\frac{\mathrm{d} p}{\mathrm{~d} t}\right|_{\text {Interactions }}\right) n\right)+q_{w} .
$$

The first term in the right-hand side describes the diffusive escape, the second term the stochastic reacceleration in turbulence, the third term is a loss term accounting for the adiabatic decompression of the cosmic ray density in the expanding medium as well as the losses due to the Coulomb interactions and to the production of pions [23], while the last term corresponds to the background injection at the wind termination shocks. The transport equation 7 is solved numerically in between supernova explosions, while when a supernova explodes, the reacceleration of the particles is computed using the nonlinear framework discussed above.

Table 1 summarises all the parameters of the model. The most important ones are the initial number of stars $N_{*}$ and the efficiency of turbulence generation $\eta_{T}$. For the latter we take a fiducial value of $30 \%$ assuming equipartition between thermal, non thermal and turbulent energy in the superbubble (e.g. [24]).

\section{Results}

\subsection{Timescales}

Figure 1 displays the timescales of the relevant physical processes discussed in the previous section. One notices that for a standard cluster of 100 stars, it is important to account for the feedback of cosmic rays onto the turbulence in order to obtain reliable results. Eventually, the evolution of the low energy bands of the cosmic ray distribution function is driven by the stochastic acceleration while the high energies are modulated by the escape. Around $\mathrm{GeV}$ energies, there exist a narrow band where supernova remnants are able to sustain all physical processes. This is the only 


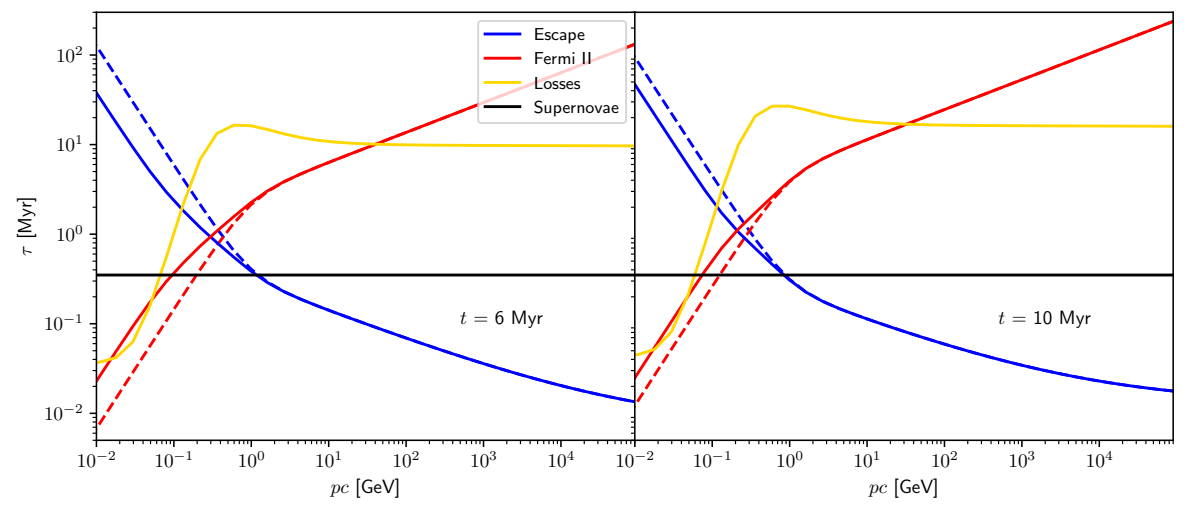

Figure 1: Timescales of escape, stochastic acceleration and losses compared to the average time interval between supernovae for a cluster of 100 massive stars, computed in the test-particle regime (dashed lines) or accounting for the cosmic ray feedback (solid lines).
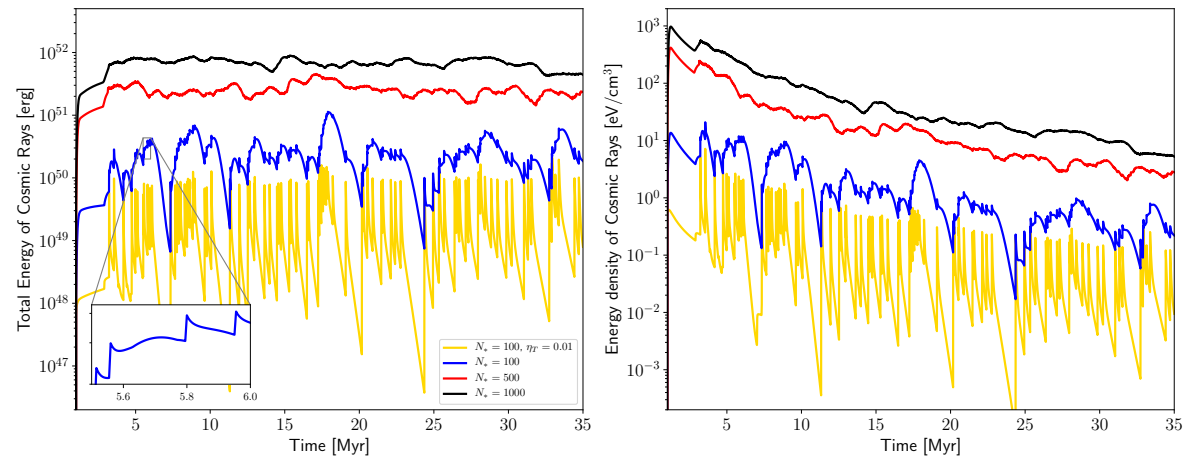

Figure 2: Total energy of cosmic rays (left) and cosmic ray energy density (right) in typical superbubbles for various numbers of stars.

band where the particle spectrum is expected to be stationary. In more massive clusters, the time interval between supernovae is lower and this energy band is larger.

\subsection{Energetics}

Figure 2 shows the evolution of the total energy of cosmic rays as well as the cosmic ray energy density inside the superbubble, from a standard cluster $\left(N_{*}=100\right)$ to a very massive cluster $\left(N_{*}=1000\right)$ such as that found in the galactic centre. While the production of cosmic rays is rather stationary for clusters containing initially several hundreds massive stars, it becomes more intermittent for typical clusters of 100 massive stars, in particular if the turbulence is not efficiently generated, as shown by the yellow curve. In this case, the particles are not confined inside the acceleration region and the superbubble somehow reduces to a collection of individual supernovae.

As shown in the zoomed-in window, the acceleration of particles proceeds in two steps: first a supernova remnant accelerates particles. Then high energy cosmic rays quickly escape, meanwhile the turbulence slowly reaccelerates low-energy particles. When most of the low-energy particles have been transferred to intermediate energy bands, the escape dominates again until another 

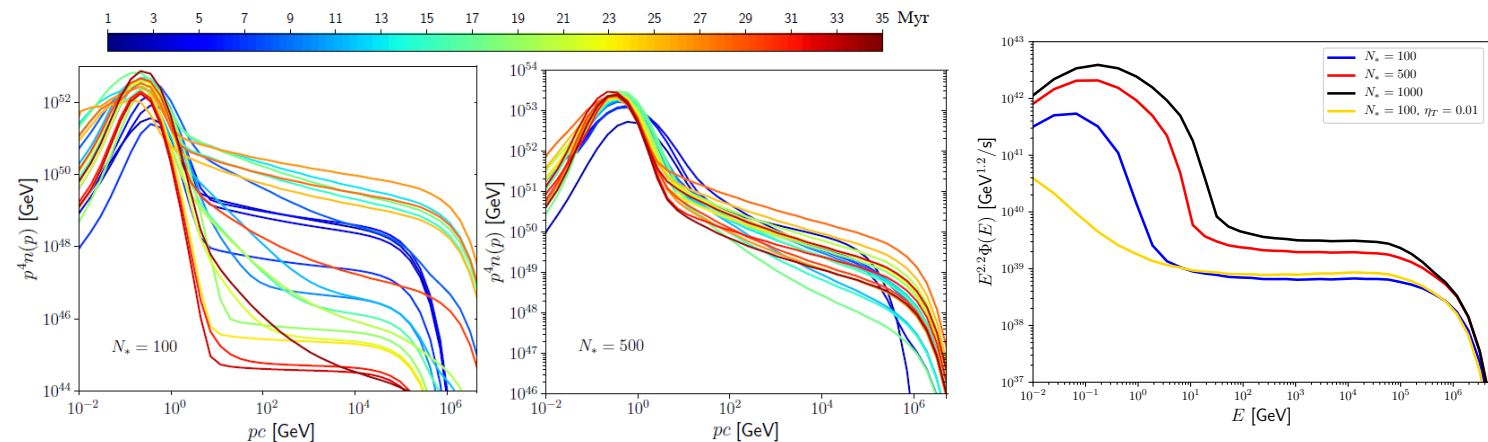

Figure 3: Cosmic ray spectra at various times for a typical cluster of 100 massive stars (left), and 500 massive stars (center). The right panel displays the average escaping fluxes.

star starts its Wolf-Rayet phase and eventually explodes. This acceleration mechanism efficiently converts the mechanical power of the stars into non-thermal particles, either directly through shock waves or indirectly through turbulence. This results in high energy densities $\left(10-100 \mathrm{eV} / \mathrm{cm}^{3}\right)$, as shown in the right panel of Figure 2.

\subsection{Spectra}

The left and middle panels of Figure 3 display examples of typical cosmic ray spectra inside superbubbles at different times. As anticipated in Section 3.1, the emission is intermittent at low momenta because of the efficient stochastic reacceleration process and at high momenta because of the efficient escape. A steady cosmic ray production only takes place at intermediate energies, around $1 \mathrm{GeV}$, for a cluster of 100 massive stars. More massive clusters display a less intermittent spectrum. Because the acceleration of high energy bands is intermittent, the wind threshold is often reached, especially for the cluster of 100 massive stars, even though the mechanical power of winds is one to two orders of magnitude below that of supernova remnants. The spectra display a typical hard/soft transition which was already noticed by Ferrand and Marcowith [8] in the test-particle regime. Because of the non-linearity of our modelling, the turbulence is regulated such that the momentum of the transition is never higher than $1 \mathrm{GeV}$. A hard spectrum up to very high energies would indeed violate the energy balance in the superbubble. Eventually, because of the transition between a hard "Fermi II bump" and a steep tail at high energies modulated by the escape, the average escape fluxes, plotted in the right panel of Figure 3, are rather steep, scaling as $E^{-2.2}$ instead of the standard $E^{-2}$ expected at isolated supernova shocks. The latter is in fact retrieved when the turbulence does not efficiently confine the particles. This further demonstrates that the steepening is not due to the nonlinear reacceleration at supernova shocks, which on the contrary tends to produce concave spectra with a hard component at high energies [18]. Interestingly, the transition between the maximum momentum achieved in wind termination shocks $(100 \mathrm{TeV})$ and that achieved at supernova remnants expanding in the low density cavity (about $1 \mathrm{PeV}$ ) leads to a spectra break which resembles a knee. 


\section{Conclusion}

The production of cosmic rays is computed in a self-consistent model accounting for all relevant ingredients rederived from first principles. It is found that the stars efficiently transfer their energy into non-thermal particles by means of shock (re)acceleration as well as stochastic acceleration in turbulence. High energy densities are achieved, which call for nonlinear models taking into account the backreaction of the cosmic rays onto the shocks and the turbulence. The typical spectra are rather intermittent, displaying a typical "Fermi II bump" from the injection energy to the GeV band, and then transitions toward a steep power-law produced by the nearly stationary wind contribution as well as the intermittent supernovae modulated by the escape. Providing the level of turbulence is about a few percents, which requires the stars to transfer a few tens of percent of their mechanical power into hydromagnetic waves, the low energy particles are efficiently reaccelerated, which gives rise to steep escape fluxes typically scaling as $E^{-2.2}$, which is close to what is needed in order to account for the diffuse cosmic ray spectrum observed on Earth. Eventually, the model can be extended to account for e.g. the effect of the supershell, which is expected to enhance the confinement of the particles. Protons-protons interactions in the shell also produce gamma-ray spectra to be compared with recent observations.

\section{References}

[1] J.C. Higdon and R.E. Lingenfelter, OB Associations, Supernova-generated Superbubbles, and the Source of Cosmic Rays, ApJ 628 (2005) 738.

[2] M. Ackermann et al., A cocoon of freshly accelerated cosmic rays detected by Fermi in the Cygnus superbubble, Science 334 (2011) 1103.

[3] A. Abramowski, F. Aharonian, F. Ait Benkhali, A.G. Akhperjanian, E.O. Angüner, M. Backes et al., The exceptionally powerful TeV $\gamma$-ray emitters in the Large Magellanic Cloud, Science 347 (2015) 406 [1501.06578].

[4] J. Katsuta, Y. Uchiyama and S. Funk, Extended gamma-ray emission from the g25.0+0.0 region: A star-forming region powered by the newly found $O B$ association?, The Astrophysical Journal 839 (2017) 129.

[5] L.A. Lopez, B.W. Grefenstette, K. Auchettl, K.K. Madsen and D. Castro, Evidence of particle acceleration in the superbubble 30 doradus $c$ with NuSTAR, The Astrophysical Journal 893 (2020) 144.

[6] A.U. Abeysekara, A. Albert, R. Alfaro, C. Alvarez, J.R.A. Camacho, J.C. Arteaga-Velázquez et al., HAWC observations of the acceleration of very-high-energy cosmic rays in the Cygnus Cocoon, Nature Astronomy (2021) [2103.06820].

[7] A.M. Bykov, Particle Acceleration and Nonthermal Phenomena in Superbubbles, Space Sci. Rev. 99 (2001) 317.

[8] G. Ferrand and A. Marcowith, On the shape of the spectrum of cosmic rays accelerated inside superbubbles, A\&A 510 (2010) A101 [0911.4457]. 
[9] M. Limongi and A. Chieffi, The Nucleosynthesis of ${ }^{26} \mathrm{Al}$ and ${ }^{60} \mathrm{Fe}$ in Solar Metallicity Stars Extending in Mass from 11 to $120 \mathrm{M}_{\text {solar }}$ : The Hydrostatic and Explosive Contributions, ApJ 647 (2006) 483 [astro-ph/0604297].

[10] R. Weaver, R. McCray, J. Castor, P. Shapiro and R. Moore, Interstellar bubbles. II. Structure and evolution., ApJ 218 (1977) 377.

[11] E. Parizot, A. Marcowith, E. van der Swaluw, A.M. Bykov and V. Tatischeff, Superbubbles and energetic particles in the Galaxy - I. Collective effects of particle acceleration, A\&A $\mathbf{4 2 4}$ (2004) 747.

[12] J. Seo, H. Kang and D. Ryu, The Contribution of Stellar Winds to Cosmic Ray Production, Journal of Korean Astronomical Society 51 (2018) 37 [1804 . 07486].

[13] G. Morlino, P. Blasi, E. Peretti and P. Cristofari, Particle acceleration in winds of star clusters, MNRAS (2021) [2102.09217].

[14] P. Blasi, Nonlinear shock acceleration in the presence of seed particles, Astroparticle Physics 21 (2004) 45.

[15] P. Blasi, S. Gabici and G. Vannoni, On the role of injection in kinetic approaches to non-linear particle acceleration at non-relativistic shock waves, MNRAS 361 (2005) 907-918.

[16] D. Caprioli, P. Blasi, E. Amato and M. Vietri, Dynamical feedback of self-generated magnetic fields in cosmic ray modified shocks, MNRAS 395 (2009) 895.

[17] D. Caprioli, Cosmic-ray acceleration in supernova remnants: non-linear theory revised, J. Cosmology Astropart. Phys. 2012 (2012) 038 [1206 . 1360].

[18] T. Vieu, S. Gabici and V. Tatischeff, Nonlinear particle reacceleration by successive shocks, In preparation (2021).

[19] V.S. Berezinskii, S.V. Bulanov, V.A. Dogiel and V.S. Ptuskin, Astrophysics of cosmic rays (1990).

[20] M.K. Verma, D.A. Roberts, M.L. Goldstein, S. Ghosh and W.T. Stribling, A numerical study of the nonlinear cascade of energy in magnetohydrodynamic turbulence, Journal of Geophysical Research: Space Physics 101 (1996) 21619.

[21] K. Fang, X.-J. Bi and P.-F. Yin, Possible origin of the slow-diffusion region around Geminga, MNRAS 488 (2019) 4074 [1903.06421].

[22] J.A. Eilek, Particle reacceleration in radio galaxies., ApJ 230 (1979) 373.

[23] K. Mannheim and R. Schlickeiser, Interactions of cosmic ray nuclei, A\&A 286 (1994) 983 [astro-ph/9402042].

[24] M. Gallegos-Garcia, B. Burkhart, A.L. Rosen, J.P. Naiman and E. Ramirez-Ruiz, Winds in Star Clusters Drive Kolmogorov Turbulence, ApJ 899 (2020) L30 [2006 . 14626]. 